disease whatever, and we cannot wonder that their impaired, imperfectly developed frames, their course of life, and uncleanliness, should promote phagedenic ulceration, and give it an unusually severe character."* I cannot here avoid briefly alluding to two cases which came under my own notice, as illustrating this very important subject-the combination of morbid actions with morbid states of constitution. One of the cases was that of a woman who was an out-patient at the Ancoats Dispensary; she had long been suffering from a syphilitic taint, which appeared to resist every attempt to relieve it. She had been treated with mercury, iodide of potassium, bark, ammonia, opium, morphia, \&c., but fresh ulcers repeatedly broke out on her legs, which had a phagedenic character, and extended to a very formidable size. Her arms were covered with crusts, like rupia. The cartilage of the nose had been destroyed before I saw her. She resided in a cellar in Fawcett-street, and evidently was in a state of poverty. The case seemed to me one which would be greatly benefited by a more generous diet, and, at my frequent solicitation, she made application to the overseers, and was eventually admitted into the workhouse, where I subsequently met with her in walking through the wards with the surgeon, and was greatly astonished to find her quite restored to health. The ulcers were all healed, and she was becoming fat. The other case had a less favourable termination. A young woman, aged twenty-three, who was a dress-maker, was the subject of a large sloughing chancre, which had existed for a long period previous to her applying for medical advice, and had destroyed the nymphx, and produced great destruction of the external parts. I directed her to have a poultice applied, and gave her a mixture containing bark and ammonia, with opium. Under this treatment the sore became cleaner, but did not seem disposed to heal. The skin was affected with rupia, as in the preceding case. There was a slight huskiness in her voice. When the sore had cleaned, a little attempt at repair was visible ; I prescribed a pill containing two grains of blue pill, with two of the iodide of potassium, to be repeated night and morning. By this means the chancre became considerably less. I then substituted sarsaparilla and the iodide of potassium, and finally bad recourse to bark and quinine. She now complained of a sore throat, and in spite of every application which could be used, it continued to increase, the tonsils and soft palate sloughed, and a foul offensive ulcer began to occupy the back of the throat. I wished to have her removed, and to procure her a more generous diet, but she would not consent, and it was impossible to obtain for her, at home, the attention and conveniences she required. The case went from bad to worse. I called several medical friends into consultation, but the girl was obstinate; her diet was bad, her constitution bad, and her habits irregular. She took poor wine, and had no meat. Her health now rapidly gave way; the legs swelled; the throat became more and more offensive; the roice was nearly gone; the right lip and cheek were paralyzed, and wie cheek swollen, as in cancrum oris. She had for some time been scarcely able to take any food, owing to the ravages of the ulcer. At last she died, and it was a happy relief to herself and her friends. This case strongly impressed me with the importance of considering diseases with a due regard to the states of constitution in which they occur, and the disorders which coexist with them.

Amongst other constitutional peculiarities we mentioned an excessive mobility of the system, which we thought to be connected with a certain molecular constitution, more especially prominent in the nervous centres. Now it might be conceived that a high mobility of the body would be connected with a delicacy of structure, which would render the body particularly susceptible of morbid action; but this is not in reality true, at least as regards the more important lesions of the body. What may be called the bysteric habit seems rather to exalt the perception of dera jgement than to render the body more easily prone to structural disease. From this circumstance, concomitant disorders are the means of discovering to us this latent peculiarity of constitution. It cannot, then, be a matter of wonder that disorders of the menstrual functions, at all times exercising great influence on the nervous system, should, under these circumstances, exert an inordinate influence. When the sensitive frame is called upon to perform new functions, for the proper discharge of which it is ill prepared, its peculiarities beccme exaggerated, and a morbid reaction is produced by the imperfect and capricious manner in which they are executed. Hence, with the approach of puberty, arise many anomalous affections, which are called hysteric, and often correctly referable to menstrual derangements. In many instances, hysteric phenomena seem connected with irritation of the spine, but whether this is a primary or secondary condition of disease is at least doubtful in the present state of knowledge.
It is probable that the coincidence of bronchitis with the hysteric constitution gives rise to some of those curious affections of the respiratory organs which are occasionally met with, and which have been commonly designated as nervous coughs. The affecticn is characterized by a shrieking or barking sound occurring in violent paroxysms, and induced by the slightest causes of excitement. The least deriation from health may be said to startle the nervous system, owing to the high susceptibility of disturbance which exists, just as the mind is alarmed by the most trifling occurrences. The patient, no donbt, really feels the greatest inconvenience or pain; in fact, there is a high appreciation of derangement.

In Sir Benjamin Brodie's Clinical Lectures on Hysteria, be states, "that in a great number of instances local hysterical symptoms appear to be connected with some accidental injury, generally a very slight one; and they are then especially liable to be misunderstood, and mistaken for something very different from what they really are."* He enumerates many instances in which an injury preceded neuralgic pains, and painful local complaints, the habit, of course, being hysterical. In some eases he found it succeed the operation of venesection, and in others injuries to the head. One ease occurred from a prick of the finger, "which was immediately followed by pain in the course of the median nerve, and on the following day the fore arm was fixed by a muscular contraction at a right angle with the arm." He relates another instance of a female, who was admitted into St. George's Hospital on account of a simple fracture of both bones of the fore arm. This was succeeded by a painful neuralgic affection of the axilla and side of the head and neck, increased by the slightest motion; the arm united as under ordinary circumstances, and the nervous symptoms subsided gradually after many weeks. The same person, it appears, had previously suffered, in a somewhat similar manner, from a slight injury of the ankle. From these and other cases, it seerns probable that many of the types and diversified forms in which hysteria is manifested, may be determined by the supervention of different morbid actions, which receive their exaggerated character from the hysterical habit in which they are found. We may conceive that where the suscep tibility is highly augmented, slight degrees of irritation may be sufficient to produce symptoms which are ordinarily only the result of serious lesions. At the same time there is still much that is inexplicable in the occurrence of local hysterical phenomena ; for many disordered actions, in hysterical subjects, will pursue their course without displaying any of the peculiarities of the accompanying diathesis, whilst others seem, if I may be allowed the expression, to solicit an undue degree of attention on the part of the nervous system. The manner in which hysteria mixes with, and modifies, various complaints incident to females, deserves a careful consideration. There are, probably, very many disorders not considered hysterical, which partake of that character in some stages of their progress.

Broughton, near Manchester, Sept. 4th, 1845.

\section{ON THE POTATO DISEASE.} By ANdrew URE, M.D., F.R.S.

THE vague and contradictory statements concerning the nature of this calamitous visitation of Providence. as well as the directions for the treatment and preservation of the tubers, generally impracticable and preposterous, which have recently issued in vast variety from the press, do little honour to economic chemistry. It is needless to notice all the notions and schemes which have either officially or spontaneously been projected. Only two of these deserve comment; the first, as coming from a great master in science, the second, as emanating from the Irish commissioners.

Professor Liebig imagines the essence of the disease to consist in the conversion of the alhumine, a usual constituent of healthy potatoes, into caseine, a principle which, by its great instability of composition, is supposed to cause the potato to putrefy rapidly. $I$ have subjected this opinion to the test of experiment. Perfectly sound potatoes, as also diseased ones, were sliced or grated, and separately digested in a very dilute alkaline ley at a blood heat. The infusions, when cool, being filtered and faintly acidulated with dilute acetic acid, afforded respectively a like proportion of caseine-looking flakes. It would thus appear, from this mode of testing, as prescribed by M. Dumas in the seventh volume of his "Traité de Chimie," that sound potatoes contain as much caseine as unsound. 
Professor Liebig's plan of preserving diseased potatoes is founded on the above notion, and consists in cutting them into slices one quarter of an inch thick, and steeping them twenty-four or thirty-six hours in dilute sulphuric acid. On this proposal I need make no comments, as it has no chance of being practised beyond the precincts of Giessen.

In the Pharmaceutical Journal for October last, I inserted a few observations on diseased potatoes, chiefly with the view of showing, that till the putrefactive stage commences, the potato had the same acidulous reaction as in the sound state, but that then a portion of ammonia made its appearance, as was proved by its alkaline action on litmus paper, and by its coming over in distillation. That brief notice was written while $I$ was at a distance from home on professional business, and where I had no means of prosecuting my experiments. At my first period of leisure since, I resumed my inquiries, and have obtained certain results which may probably be found useful, as well as interesting. Before entering into a detail of them, I shall shortly describe the constituents of sound potatoes, according to the must authentic analyses. Their average composition in 100 parts, according to Einhof and Lampadius, is-fibrous matter, 7 ; starch, 15 ; vegetable albumen, 1 ; gum, acids, and salts, 3.5; water, 75. Besides these principles, Vauquelin, by his older and more minute analysis, discorered the following in minute quantities:-crystallizable asparagin ; an azotised substance resembling gum; a resinous matter emitting an agreeable odour when heated; an extractive matter which blackens in the air ; citric acid; citrates and phosphates of potash and lime.

The fibrous matter of potatoes is not of the same species as that of woody roots, but consists of a substance analogous to starch, which swells in water, becomes transparent, dissolves for the most part in dilute sulphuric acid, and affords gum and sugar only by the action of concentrated sulphuric acid. In some of Einhof's experiments, the gummy matter which remained after evaporating the potato juice was saccharine, but he ascribed the formation of his sugar to an alteration produced on a portion of the gum in the conrse of analysis. Neither he, nor Lampadius, nor even Vauquelin, found sugar in sound potatoes. There can be no doubt, however, that, from several causes, potatoes may become sweet-tasted, indicating that part of their starch is saccharified. Thus, by exposing them to repeated alternations of temperature, a few degrees below and above that of melting ice, the formation of sugar is so much promoted, that they grow soft with the production of a syrup of so rich a nature, that it will not permit the potatoes to solidify even when cooled several degrees below $32^{\circ}$. Fahrenheit. This curions transmutation seems to depend, at least in its origin, upon a vital reaction; for when they are frozen very rapidly, no sugar is formed either during or after their thawing; but, on the contrary, potatoes so treated afford more starch than otherwise.

The nutritious quality of potatoes resides chiefly in the starch, fibrine, and albumine; the latter being essential to the formation of blood. Boussingault rates the feeding of a cow for twentyfour hours at fifteen kilogrammes (about thirty-three pounds avoirdupoise) of potatoes, and the quantity of azote in them at fifty grammes, or $\frac{1}{300}$ th part of their weight. This would give $\frac{1}{75}$ th part of azote in dried potatoes. Now, since these contain four times as much albumine as the moist ones do, we shall have four per cent. of albumen in them, according to the above analysis, which four parts contain the whole of the azote in 100 parts of dried potatoes, amounting to $\frac{1}{7}$ th, or one and one-third per cent. But 100 parts of albumen, according to Dumas and Cahours, contain $\mathbf{1 5 . 7 5}$ of azote, and four will therefore contain only 0.63 , being about one-half the proportion of azote assigned by Boussingault. Having, in the course of my frequently recurring analyses of guano, contrived a method of determining its proportion of azote, even to $\frac{1}{100}$ th of a grain, or half a milligramme, I have recently had recourse to this method in the examination of potatoes. When a portion of these, in a dry, pulverulent state, was subjected to ignition in contact with hydrate of soda and lime, 0.579 of azote were obtained from 100 parts of the potato; a number which accords well with that deduced from Dumas and Cahours' resulte, as applied to the proportion of albumine in potatoes, an accordance which seems to justify my determination. Potato, in its ordinary moist state, will hence contain about 0.015 of azote, or one and a half parts in a thousand. To this element, and the animal principles into which it enters, the nutritive quality of potatoes is to be ascribed, while their starch and starchy fibrine afford the fuel of animal temperature.

In the diseased potatoes, a portion of the starch is transformed into sugar, and of the albumine into an acrid offensive brown substance. If sueh tubers as are characterized by brown spots in the interior, and a thickened brown skin, bath composed of fungous fibres, be grated or sliced, and exposed to pressure, either alone or with a little tepid water, the juice obtained will be found to have a mawkish sweet taste, followed by a sense of pungency on the tip of the tongue. If some of this juice be mixed with a little of Trommer's grape-sugar test, (an alkalized solution of sulphate of copper,) this blue-coloured mixture will change into a bright orange hue, slowly, in the cold, but rapidly on the application of a gentle heat, with a deposit of protoxide of copper. By means of a modification of that test, described by me in the Pharmaceutical Journal for July, 1842, I have ascertained the existence of about five per cent. of saccharine matter in diseased potatoes ; yet by the same re-agent, which is sensible to $\frac{1}{100}$ th of a grain of sugar, I could observe none of it in perfectly sound potato. After satisfying myself, in this way, as to the presence of sugar in diseased potatoes, I proceeded to verify the fact hy placing their expressed juice, as also their infusion, in contact with a little yeast, at a fermenting heat of from $80^{\circ}$ to $90^{\circ}$ Fahrenheit, and watched the resulting phenomena. A fermentative action soon began, and in an hour or two became so brisk as to throw up a thick, creamy froth, like that occurring with smallbeer wort. At the end of thirty-six hours, the liquor having considerably diminished in specific gravity, was subjected to distillation, and yielded alcohol equivalent to about four per cent. of sugar in the potato. In order to obviate any chance of fallacy in these results, I requested $\mathrm{Mr}$. Scanlan to repeat my fermentation experiments on a somewhat larger scale than mine, with diseased potatoes found in his own neighbourhood, at the extremity of London, opposite to my residence. He has accordingly made several similar researches, with like results, of which I shall give the particulars of one only. 'Two and a half pounds of the expressed juice of such potatoes as are now used solely for feeding cattle, corresponding to three and one-third pounds of the entire tubers, were mixed with a small portion of yeast, and set to ferment in half-filled bottles. The action soon became so vigorous that the barm rose to the brim of the bottles. At the end of thirtysix hours, the liquor, having become nearly tranquil, was distilled, and afforded 5000 water-grain measures of an alcoholic liquid, of specific gravity of 0.988 , containing, by 'Tralle's table, eight per cent. of absolute alcohol, equivalent to sixteen of sugar. $16 \times 50$ $=800$ grains, may be regarded as the quantity of sugar indicated by this experiment in three and a half pounds of the diseased potatoes, or in 23,333 grains, being nearly three and a half per cent. In other samples I found, as above stated, results indicating considerably more sugar, the proportion being very variable according to the state of the disease. The vinous spirit produced is by no means disagreeable in taste or flavour, and may be easily rectified into excellent alcohol, fit for every purpose of arts, manufactures, and pharmacy. Were it not for the oppressive laws of the Excise, sufficient alcohol might thus be obtained this season for the uses of a temperate people, reserving an equivalent portion of grain from the whisky manufacture for their sustenance. The residual cake of the diseased potato is well adapted for feeding cattle, the morbid juices having been separated, and it may be so dried as to keep unchanged for a moderate length of time.

In all the diseased potatoes which I have examined with the microscope, the fibres of a fungus called botrytis from its grapelike form- or of one called uredo tuberosum, may be observed ramifying round the cells which enclose the starchy corpuscles. Now these plants, however minute, are not self-generated, but must be produced by some seminal impregnation transported by the atmosphere, and peculiarly adapted to fructify upon the solanum tuberosum. I would hence conclude, that the potato disease is a peculiar vegeto-pestilence, diff used generally through the atmosphere, whose ravages have been favoured by the sunless humidity of the last season as the predisposing, but not as the exciting, cause. The proximate cause again, in medical language, or the essence of the morbid state, is the fungous inmate of the tuber, from seminal impregnation of the stem, which so paralyzes the vitality of the plant, that a portion of the starch and albumen becomes decomposed. This vegetable distemper, like that of the cholera, while general in its diffusion, is determined to particular localities and plants by certain predisposing causes; yet it is independent of these, having occurred in many regions where such causes did not materially operate. Whether it will recur, no human being can predict; meanwhile, it reads a great and solemn lesson to the rulers of states, never better expressed than in Virgil's well known rerse-

"Discite justitiam moniti, et non temnere diros;"

which may be translated, "Beware of obstructing the free supply of food to your people."

Many preposterous prescriptions have been obtruded on the public eye as to the best method of preserving the diseased potatoes from putrefaction. The above researches show the existence of a highly fermentable saccharine and albuminous matter in them, which becomes rapidly operative by contact with air and 
moisture. Care should therefore be taken to keep their skins entire, so as to exclude the atmospheric oxygen and humidity. It is well known that the sugar in ripe grapes undergoes no change while the skin is entire, but the moment this is pricked, the grapes begin to ferment, and speedily spoil. No plan is therefore more to be deprecated than that of slicing and mashing potatoes. They should be placed in an atmosphere kept by chemical means in a state of extreme dryness, which may be easily and cheaply effected by piling them upon a bed of brushwood, dry turf, or straw, interspersing through the pile unslaked lime coarsely bruised, and covering the pile thoroughly at the sides and on the top from the external elements. Since unslaked lime absorbs greedily one-third of its weight of moisture, it will bring the air in the spaces between the tubers into a perfectly arid state-a condition in which no decomposition of the substance can possibly take place. On the same principle, highly-polished steel articles may be kept for any length of time without tarnishing in our humid climate, provided a basin with lumps of unslaked lime be enclosed in the case or chest containing them. Slaked lime, on the contrary, being saturated with water, has no power of desiccation, but acts only by its causticity in farouring the destruction of all vegetable and animal matter.

Charlotte-street, Bedford-square, Dec. 9th, 1845.

\section{CASE OF POISONING WITH THE BICHLORIDE OF MERCURY.}

By Wruliam Bird Herapath, M.B. Lond., Bristol.

THe following case of poisoning by the bichloride of mercury in solution, presents many interesting peculiarities. It occurred recently in the practice of my partner, Mr. S. Bryant, and myself; we watched it closely throughout, but, from peculiar eircumstances, were unable to render the patient all the assistance we wished:-

\section{SUMAIRY OF THE CASE.}

1st day.-An attenuated epileptic patient swallowed a fluid containing a scruple of bichloride of mercury in solution. Vomiting came on in two minutes afterwards, and diarrhoea in fifteen, which continued during an hour. Stomach-pump used, and albumen injected.

2nd.-Diarrhœa continued; vomiting ceased; system rallied from the shock.

3rd.-Diarrhoea ceased; suppression of urine, and ptyalism came on.

4th.-Ptyalism and suppression continued.

5th.-Diarrhœa, dysentery, and tenesmus came on; great prostration and violent pain; sloughing of the gums, lips, and tongue.

6th.-Rallied; tenesmus continued; febrile excitement came on in the evening.

7 th-Excitement of the vascular system continued.

8th.-Excitement passed off; coma came on; convulsions

followed. Jactitation and trembling ensued.

9th.-Coma increased; convulsions more frequent; prostration ; congestion of the lnngs; respiration became embarrassed. 10th.-Death occurred early.

Autopsy.-Esophagus healthy; stomach inflamed; mucous membrane injected and extensively softened; some blood effused; vascularity of small intestines; cæcal extremity of ilium almost gangrenous; cæcum most highly inflamed, gangrenous, and sloughing; sigmoid flexure of colon and rectum also ulcerated, sloughing, and much inflamed; lungs congested, labular pnenmonia of the left.

\section{REVIEWS.}

Medical and Physiological Problems. By Wrumam Griffin, M.D., and Daniel Grifrin, M.D. Sherwood, Gilbert, and Piper. 1845. pp. 356.

ThIs volume, the work of two physicians practising in Limerick, is certainly a highly respectable performance. It is gratifying to see two such men, living in a remote city, animated by a sincere love of the profession of medicine, and seeking above all things to arrive at true conclusions, combining their thoughtful experiences into a record which may be useful to others walking in the same path, but with feebler steps than their own. The severer edge of criticism is disarmed by a contemplation of the difficulties attending the attempt to advance medical science under such disadvantageous circumstances; for these essays do not seek merely to follow, but to lead, in the points on which they dwell. The work contains thirteen essays or problems, some of which are physiological and some practical. Of these, Dr. W. Griffin is the author of principles of treatment in enteritis; diagnosis between nervous and inflammatory affections; diagnosis of abdominal inflammations ; spinal irritation ; sudden death in jaundice; crowing inspiration; consciousness; therapeutic effects of opium; treatment of hæmoptysis ; treatment of rheumatism. Dr. D. Griffin is the author of papers on bleeding in diseases of the brain ; and on the law of visible direction in optics; while the two gentlemen are joint authors of the last paper, on the application of mathematics to the science of medicine. We may observe, that the principal writer, Dr. W. Griffin, appears the most practical, Dr. D. Griffin the most speculative; and, we may remark, that we prefer the diagnostic and therapeutic papers to the rest. When either is writing on clinical points or things passing under his own observation and reflection, there is much that is valuable, but when they pass to the later knowledge and hypotheses of the day, the misfortune of their provincial position becomes apparent. Evidently their minds are of sufficient calibre to grasp all we know, but in some instances they are wanting in new material to work upon.

In the paper on enteric inflammation, the claims of opium are strongly enforced. This medicine is put in opposition to purgative medicines in this disease-vascular depletion being practised in either case. Those who advocate the purgative treatment rely on the diminution of the abdominal and general circulation by the increased secretions and the removal of feces, as more than sufficient to counterbalance the irritation of the purgative itself; those who support the opiate treatment, consider the repose of the intestine under its influence to be more than a compensation for the irritation caused by fæcal accumulation. Another item in the calculation is, that opium, by diminishing suffering, obviates, in some measure, the extremely depressing influence of abdominal pain on the heart's action - a point worth considering in all painful diseases of the bowels. Dr. W. Griffin is decidedly in favour of the opiate treatment in the majority of cases.

In the remarks on the diagnosis of nervous and inflammatory diseases of the thorax and abdomen, Dr. W. Grifin strongly insists on the importance of examining the spinal column. He maintains, that in neuralgic pains simulating pleuritis, peritonitis, \&c., there is evident tenderness of that portion of the spinal column corresponding to the disturbed organ, while this is wanting in inflammatory cases. He even goes so far as to say that this "is almost the only single symptom upon which a young practitioner can rely without danger." Extended experience on the part of other physicians will be required to verify this sanguine assertion.

The paper on bloodletting in diseases of the brain condemns large bleedings because of the uncertain symptomatology of cerebral affections, and particularly where extensive disease exists, because they injure the reparative processes, and in severely painful affections, as such cases generally terminate in exhaustion. 'These remarks are, of course, intended to apply to apoplectic and epileptic cases rather than acute inflammation.

The remarks on sudden deaths in jaundice refer to the supervention of fatal coma in this disease. The question is argued, whether, in such cases, the hepatic or the cerebral affection is the idiopathic disease. The author leans to the conclusion, that the brain becomes affected, owing to the accumulation of azote and carbon in the blood. The cerebral affection in jaundice certainly bears some resemblance to that occurring in suppression of the renal secretion, from the presence of urea in the blood, except that in jaundice the brain is only occasionally affected. Four cases are given, two of them ending fatally, the other two recovering; these were treated by active purgation.

In the paper on optics, Dr, D. Griffin contends strongly against the theory of the law of visible direction supported by Sir David Brewster, and upholds his opinion by a very cogent train of reasoning and calculation. Other eminent scientific observers have made objection to the common theory, and there can be no doubt but that the point at present remains a questio vexata in optics. 